\title{
THE VOLUME OF THE MODULI SPACE OF FLAT CONNECTIONS ON A NONORIENTABLE 2-MANIFOLD
}

\author{
NAN-KUO HO AND LISA C. JEFFREY
}

\begin{abstract}
We compute the Riemannian volume of the moduli space of flat connections on a nonorientable 2-manifold, for a natural class of metrics. We also show that Witten's volume formula for these moduli spaces may be derived using Haar measure, and we give a new proof of Witten's volume formula for the moduli space of flat connections on a 2-manifold using Haar measure.
\end{abstract}

\section{INTRODUCTION}

In W] Witten defined and computed a volume on the moduli space of gauge equivalence classes of flat connections on a 2-manifold, using Reidemeister-Ray-Singer torsion (see e.g. [Fr]). When the 2-manifold is orientable, Witten proved that this volume is equal to the symplectic volume on the moduli space. However, when the 2-manifold is not orientable the moduli space does not have a symplectic form, so the interpretation of this volume has been unclear.

The moduli space of gauge equivalence classes of flat connections on a nonorientable 2-manifold turns out to be a Lagrangian submanifold of the moduli space of gauge equivalence classes of flat connections on the orientable double cover. In this article we compute its Riemannian volume for a natural class of metrics on the 2-manifold. The dependence on the choice of metric is discussed in Remarks 1 and 2 below.

The layout of this article is as follows. In Section 2 we compute the Riemannian volume of the moduli space of flat connections on a nonorientable 2-manifold (exhibited as the connected sum of a Riemann surface with either one or two copies of $R P^{2}$ ) using a metric derived from a particular class of metrics on $R P^{2}-\{$ disc $\}$. In Section 3 we give a new proof that Witten's formula for the volume of the moduli space of flat connections on 2-manifolds (for nonorientable surfaces with no boundary and orientable surfaces with one boundary component) arises from Haar measure.

The first author was partially supported by grants from OGS and OGSST.

The second author was partially supported by a grant from NSERC. 
Acknowledgement: This article comprises part of the Ph.D. thesis of the first author, under the supervision of the second author. Both authors would like to thank Eckhard Meinrenken and Chris Woodward for useful conversations and insightful advice.

\section{Metrics on the Moduli SPACE}

2.1. Preliminaries. By the classification of 2-manifolds [Ma , all nonorientable 2-manifolds are obtained as the connected sum of a Riemann surface with either one copy of the real projective plane $R P^{2}$ (denoted $P$ ) or two copies of $P$ (which is equivalent to the connected sum with a Klein bottle $K$ ).

Given a Riemannian metric on the Möbius strip $P \backslash D$, we obtain a Riemannian metric on the connected sum $\Sigma \# P$ or $\Sigma \# P \# P$, where $\Sigma$ is an oriented 2-manifold equipped with a Riemannian metric. We assume that a Riemannian metric has been chosen on $\Sigma \backslash D$.

We view $P \backslash D$ as formed by gluing together two sides of equal length of a triangle $\triangle$. We define a metric on $P \backslash D$ by endowing $\triangle$ with a Riemannian metric in which two sides are of equal length and using geodesic polar coordinates about one vertex of the triangle. In such coordinates $(\rho, \sigma)$ (where $\rho$ is the arc length from $p$ and $\sigma$ is the angular variable) the metric takes the form ( $\mathrm{dC}$. Section 4.6)

$$
d s^{2}=d \rho^{2}+g_{22}(\rho, \sigma) d \sigma^{2}
$$

We now assume that the triangle $\triangle$ is a geodesic triangle. In geodesic polar coordinates, the Hodge star operator is then

$$
\begin{gathered}
* d \rho=\sqrt{g_{22}} d \sigma \\
* d \sigma=-\frac{1}{\sqrt{g_{22}}} d \rho
\end{gathered}
$$

We make the assumption that $g_{22}=g_{22}(\rho)$ depends only on $\rho$ and is independent of $\sigma$. This assumption is satisfied by the three important examples of metrics with constant scalar curvature:

(1) Spherical metric with scalar curvature $+1: g_{22}(\rho)=\sin ^{2}(\rho)$

(2) Euclidean metric (with scalar curvature 0): $g_{22}(\rho)=\rho^{2}$

(3) Hyperbolic metric with scalar curvature $-1: g_{22}(\rho)=\sinh ^{2}(\rho)$

We can consider two triangles shown in Figure 1. We use geodesic polar coordinates at the vertex $x_{0}$. The curves $\gamma_{1}$ and $\gamma_{2}$ are radial geodesics with constant $\sigma$. The curve $\gamma_{3}$ is the geodesic joining the two vertices $x_{1}$ and $x_{2}$. The curve $C_{1}$ is the curve $\rho=$ constant between $x_{1}$ and $x_{2}$; it is not a geodesic. The triangle $\triangle$ is bounded by $\gamma_{1}, \gamma_{2}, \gamma_{3}$; 
it is a geodesic triangle. The triangle $\triangle_{0}$ is bounded by $\gamma_{1}, \gamma_{2}, C_{1}$. We can find the integral over the triangle $\triangle_{0}$ bounded by $\gamma_{1}, \gamma_{2}, C_{1}$ where $C_{1}$ is the curve specified by the equation $\rho=L=$ constant.

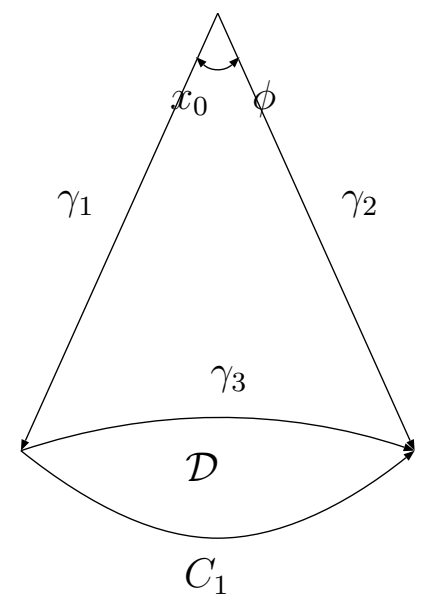

Figure 1. Triangles $\triangle$ and $\triangle_{0}$

Let $G$ be a compact connected Lie group; the moduli space of flat connections on $\triangle$ modulo based gauge transformations (i.e. gauge transformations which take the value identity on the three vertices) can be identified with $G \times G$. The moduli space of flat connections on $P \backslash \mathcal{D}$ modulo gauge transformations which are trivial at one point on the boundary (corresponding to the vertices of the geodesic triangle) is equivalent to the subspace of the moduli space of flat connections on the geodesic triangle (modulo based gauge transformations) which correspond under an orientation reversing map which identifies two sides of the triangle. This is $\operatorname{Hom}(\pi, G)$ where $\pi=\pi_{1}(P \backslash D)$, in other words

$$
\operatorname{Hom}(\pi, G)=\left\{(u, v) \in G \times G \mid u v^{2}=1\right\} \cong G .
$$

Here $v$ is the holonomy along $\gamma_{1}$ and $-\gamma_{2}$, while $u$ is the holonomy along $\gamma_{3}$.

On the space of flat connections on $\triangle$, there is a metric

$$
<a, b>=\int_{\triangle} \operatorname{Tr}(a \wedge * b)
$$

where $*$ is the Hodge star operator on differential forms over $\triangle$. We will study the corresponding metric on $G \times G$. Define the metric $<$, > at a flat connection $A$ corresponding to a point $p$ in $G \times G$ to be

$$
<d_{A} \xi, d_{A} \eta>=: \int_{\triangle} \operatorname{Tr}\left(d_{A} \xi \wedge * d_{A} \eta\right)
$$


where $\xi(\cdot)$ and $\eta(\cdot)$ are $\mathfrak{g}$-valued functions on $\triangle$, so $d_{A} \xi$ and $d_{A} \eta$ represent elements of the tangent space to $G \times G$ at $p$. Here, $\operatorname{Tr}(\cdot)$ represents the ad-invariant inner product on the Lie algebra.

In the case when $G$ is abelian, the holonomy of $d \xi$ along $\gamma_{1}$ is

$$
H o l_{\gamma_{1}} d \xi=\exp \int_{\gamma_{1}} d \xi=\exp \left(\xi\left(\rho=L_{1}, \sigma=0\right)-\xi(\rho=0, \sigma=0)\right),
$$

and its holonomy along $\gamma_{2}$ is

$$
H_{o l} l_{\gamma_{2}} d \xi=\exp \int_{\gamma_{2}} d \xi=\exp \left(\xi\left(\rho=L_{2}, \sigma=0\right)-\xi(\rho=0, \sigma=\phi)\right) .
$$

We will study these holonomies once we have determined $\xi$.

From now on, we only consider the inner product at $A=0$. The reason is that the metric $\langle a, b\rangle=\int \operatorname{Tr}(a \wedge * b)$ is ad-invariant (where * denotes the Hodge star operator), and since the gauge action at the infinitesimal level is the adjoint action, this metric is invariant under the action of the gauge group and in particular under the action of the based gauge group. Since the space $\triangle$ is contractible, any flat connection is gauge equivalent to the trivial connection on a triangle. In other words all infinitesimal flat connections - elements of the tangent space of the space of flat connections modulo based gauge transformations - can be expressed as $d \xi$ for some infinitesimal gauge transformation $\xi: \triangle \rightarrow \mathfrak{g}$. So we need only consider the inner product at $A=0$.

We want to associate a norm $<d \xi, d \xi>$ where $\xi \in \Omega^{0}(P \backslash D) \otimes \mathfrak{g}$. This will be done by defining

$$
<d \xi, d \xi>=: \int_{\triangle} \operatorname{Tr}(d \xi \wedge * d \xi)
$$

Using Stokes' theorem, this can be converted to a line integral around the boundary of the triangle if we have $d * d \xi=0$ : this follows because

$$
\begin{aligned}
\int_{\triangle} \operatorname{Tr}(d \xi \wedge * d \xi) & =\int_{\triangle} \operatorname{Tr}[d(\xi \wedge * d \xi)-\xi d * d \xi] \\
& =\int_{\partial \triangle} \operatorname{Tr} \xi * d \xi-\int_{\triangle} \operatorname{Tr} \xi d * d \xi
\end{aligned}
$$

In fact, we may assume $\xi$ satisfies

$$
d * d \xi=0 \text {. }
$$

This condition represents a transversal to the orbit of the based gauge group; our procedure is analogous to identifying de Rham cohomology classes with harmonic forms (forms $\alpha$ satisfying $d \alpha=0$ and $d * \alpha=0$ ). Note that the space $G \times G$ is isomorphic to the space of flat connections 
on $\triangle$ modulo based gauge transformations (i.e. gauge transformations which take the value 1 at the vertices). Each equivalence class may be written as $d \xi$ for some $\xi: \triangle \rightarrow \mathfrak{g}$ (which does not take value 1 at all the vertices, unless one wishes to represent the trivial flat connection).

To solve equation (17), first, let us recall some geometry. Recall that our Riemannian metric in geodesic polar coordinates is

$$
d s^{2}=d \rho^{2}+g_{22}(\rho) d \sigma^{2}
$$

where $\rho$ is the distance from a chosen point $x_{0}$ and $\sigma$ is the polar angle with respect to this point. Let $*$ denote the Hodge star operator on the 2-manifold; the star operator with respect to these polar coordinates is

$$
* d \rho=\sqrt{g_{22}(\rho)} d \sigma, * d \sigma=\frac{-1}{\sqrt{g_{22}(\rho)}} d \rho .
$$

We use the substitution

$$
u(\rho)=\int^{\rho} \frac{1}{\sqrt{g_{22}(t)}} d t
$$

and define

$$
\tau(\rho)=\exp u(\rho)
$$

The equations for the Hodge star operator become

$$
\begin{gathered}
* d u=d \sigma \\
* d \sigma=-d u
\end{gathered}
$$

We now have to solve the equation

$$
d(* d \xi)=0
$$

We have

SO

$$
d \xi=\frac{\partial \xi}{\partial u} d u+\frac{\partial \xi}{\partial \sigma} d \sigma
$$

$$
\begin{aligned}
d(* d \xi) & =d\left(\frac{\partial \xi}{\partial u} d \sigma-\frac{\partial \xi}{\partial \sigma} d u\right)=\left(\frac{\partial^{2} \xi}{\partial \sigma^{2}}+\frac{\partial^{2} \xi}{\partial u^{2}}\right) d u \wedge d \sigma \\
& =\left(\xi_{\sigma \sigma}+\xi_{u u}\right) d u \wedge d \sigma
\end{aligned}
$$

So the equation we need to solve is

$$
\xi_{\sigma \sigma}+\xi_{u u}=0
$$

Let us take a Fourier decomposition of $\xi$ in the $\sigma$ variable:

$$
\xi(u, \sigma)=\int_{\mathbb{R}} \hat{\xi}(u, k) e^{i k \sigma} d k
$$


So (10) becomes

$$
\frac{\partial^{2}}{\partial u^{2}} \hat{\xi}(u, k)-k^{2} \hat{\xi}(u, k)=0
$$

This equation (11) has the following solutions:

$$
\hat{\xi}(u, k)=C_{+}(k) \tau^{k}+C_{-}(k) \tau^{-k} .
$$

Recall that we had defined $\tau=\exp (u)$. Imposing the condition that $\hat{\xi}(u, k)$ is finite at $u=0$, we get

$$
\hat{\xi}(u, k)=C_{+}(k) \tau^{k}
$$

when $k>0$, and

$$
\hat{\xi}(u, k)=C_{-}(k) \tau^{|k|}
$$

when $k<0$. Recall that $\phi$ is the polar angle of the geodesic triangle. Let us impose the additional constraint that $\xi(u, \sigma)$ attains its maximum on two edges of the triangle $\left(\gamma_{1}\right.$ which is $\sigma=0$ and $\gamma_{2}$ which is $\sigma=\phi)$, in other words $\frac{\partial}{\partial \sigma} \xi(\rho, \sigma)=0$ when $\sigma=0$ and $\sigma=\phi$. This leads to

$$
\xi(u, \sigma)=Y \tau^{k} \cos k \sigma
$$

where $Y \in \mathfrak{g}$ is a constant and

$$
k=\pi / \phi .
$$

We compute that

$$
\begin{gathered}
\int_{C_{1}} \operatorname{Tr}(\xi \wedge * d \xi)=\int_{0}^{\phi}<\xi, \partial \xi / \partial u>d \sigma \\
=<Y, Y>k \exp k u(L) \int_{0}^{\phi} \cos ^{2} k \sigma d \sigma \\
=<Y, Y>\pi / 2 \exp k u(L) .
\end{gathered}
$$

Note that writing $\xi$ in terms of its Fourier decomposition accomplishes the same thing as solving the equation $d * d \xi=0$ by the method of separation of variables. We solved this equation in the previous subsection. Thus we obtain

$$
\xi(\rho, \sigma)=Y \tau(\rho)^{k} \cos k \sigma, k=\pi / \phi
$$

so that the maximum of $|\xi|$ is achieved on the edges $\sigma=0$ and $\sigma=\phi$ and $Y \in \mathfrak{g}$ is a variable we choose so that we can get the desired 
holonomy along the boundary. The holonomies were specified by the equations (4) and (5).

$$
\begin{gathered}
\int_{C_{1}} \operatorname{Tr}(\xi \wedge * d \xi)=<Y, Y>k\left[\tau\left(\frac{L}{2}\right)\right]^{2 k} \int_{0}^{\phi} d \sigma \cos ^{2} k \sigma \\
=<Y, Y>\left[\tau\left(\frac{L}{2}\right)\right]^{2 k} \frac{\pi}{2} \\
\int_{\gamma_{1}} \operatorname{Tr}(\xi \wedge * d \xi)=\int_{\gamma_{2}} \operatorname{Tr}(\xi \wedge * d \xi)=0
\end{gathered}
$$

Thus (referring to Figure 1) the integral over the geodesic triangle $\triangle$ bounded by $\gamma_{1}, \gamma_{2}, \gamma_{3}$ is the integral over the triangle $\triangle_{0}$ bounded by $\gamma_{1}, \gamma_{2}, C_{1}$ minus the integral over the region $\mathcal{D}$ :

$$
\int_{\triangle} \operatorname{Tr}(d \xi \wedge * d \xi)=\int_{\triangle_{0}} \operatorname{Tr}(d \xi \wedge * d \xi)-\int_{\mathcal{D}} \operatorname{Tr}(d \xi \wedge * d \xi) .
$$

This leads to

$$
\int_{\triangle} \operatorname{Tr}(d \xi \wedge * d \xi)=\int_{C_{1}} \operatorname{Tr}(\xi \wedge * d \xi)-\int_{\mathcal{D}} \operatorname{Tr}(d \xi \wedge * d \xi) .
$$

The integral over $\mathcal{D}$ is given as follows:

$$
\begin{gathered}
\int_{\mathcal{D}} \operatorname{Tr}(d \xi \wedge * d \xi)=\int_{\mathcal{D}}<Y, Y>k^{2} \tau^{2 k}\left(\frac{\cos ^{2} k \sigma}{\tau}+\frac{\sin ^{2} k \sigma}{\tau}\right) d \tau \wedge d \sigma \\
=\int_{\mathcal{D}}<Y, Y>k^{2} \tau^{2 k} \frac{1}{\tau} d \tau \wedge d \sigma \\
=<Y, Y>k^{2} \int_{0}^{\phi} d \sigma \int_{\tau\left(\gamma_{3}(\sigma)\right)}^{\tau\left(C_{1}(\sigma)\right)} \tau^{2 k} \frac{d \tau}{\tau} \\
=<Y, Y>k^{2} \int_{0}^{\phi}\left(\frac{\tau^{2 k}\left(C_{1}(\sigma)\right)}{2 k}-\frac{\tau^{2 k}\left(\gamma_{3}(\sigma)\right)}{2 k}\right) d \sigma
\end{gathered}
$$

where $\tau\left(C_{1}(\sigma)\right)$ is a constant independent of $\sigma$.

Remark 1. If $g$ is a Riemannian metric on $\triangle$ then the quantity

$$
<d \xi, d \xi>_{g}
$$

(associated to the metric $g$ ) is equal to the quantity $<d \xi, d \xi>_{g e w}$ where $w: P \backslash D \rightarrow \mathbb{R}$ is a $C^{\infty}$ function. In other words our definition of the metric $<d \xi, d \xi>_{g}$ is invariant under conformal transformations on $\triangle$. To see this, we write the metric as

$$
g=\left[\begin{array}{ll}
g_{11} & g_{12} \\
g_{21} & g_{22}
\end{array}\right]
$$


with inverse

$$
g^{-1}=\left[\begin{array}{ll}
g^{11} & g^{12} \\
g^{21} & g^{22}
\end{array}\right] .
$$

We observe that the Hodge star operator is unchanged under $g \mapsto g e^{w}$ (where $w: \triangle \rightarrow \mathbb{R})$, since $\operatorname{det} g$ transforms to $(\operatorname{det} g) e^{2 w}$ while $g^{11}$ transforms to $g^{11} e^{-w}$. The Hodge star operator for coordinates $x_{1}, x_{2}$ is

$$
* d x_{1}=g^{11} \sqrt{\operatorname{det} g} d x_{2}
$$

Hence $g^{11} \sqrt{\operatorname{det} g}$ is unchanged. Thus the norm (denoted by $\Gamma$ ) on $d \xi$ computed using metrics of the form

$$
d s^{2}=d \rho^{2}+g_{22}(\rho) d \sigma^{2}
$$

also gives the norm for metrics $g$ conformally equivalent to those of the form (19).

Remark 2. In fact in dimension 2 every Riemannian metric is locally diffeomorphic to one which is conformally equivalent to a metric of constant curvature (see [d'H] Section 3.3). This shows that all metrics on $P \backslash D$ are conformally equivalent to a metric of constant curvature, one of the three listed in (2.1), for which the norm (6) may be computed as in (16). The values of the norms are different for the three different choices of constant curvature metrics.

Remark 3. The volume of the moduli space defined using ReidemeisterRay-Singer torsion is independent of the choice of metric on the 2manifold either orientable or nonorientable. The Riemannian volume, however, is a different story. For an orientable 2-manifold, the moduli space of flat connections is a Kähler manifold, so the Riemannian volume equals the symplectic volume, and is thus independent of the choice of metric. For a nonorientable surface, Witten remarks in [W] (p. 163) that the Riemannian volume does depends on the metric. According to W] (2.38) the torsion volume and the Riemannian volume differ (in this case) by a ratio of determinants of elliptic operators (generalized Laplacians on Lie algebra-valued differential forms). Our results are consistent with this observation, and at the same time provide an evaluation of this ratio of determinants.

2.2. Hyperbolic metric on the geodesic triangle. We explore the hyperbolic case first. Note that if $\Sigma$ has genus $\geq 2$ then by the uniformization theorem it is equipped with a unique metric of constant scalar curvature -1 (in other words a hyperbolic metric). This is one motivation for choosing a hyperbolic metric on $P \backslash D$, although such a metric will be singular at one point. 
A Riemann surface with one boundary component and genus $\geq 1$ always has a hyperbolic metric with constant curvature $-1[\mathrm{~B}$. For this reason we construct a metric on $P \backslash D$ for which the boundary is a geodesic and which is hyperbolic with constant curvature -1 at all but one point. We do this by gluing the edges of an isosceles geodesic hyperbolic triangle using an orientation reversing map: this process identifies all three vertices of the triangle, and the vertex becomes the point where the metric does not have constant curvature -1 (indeed, the metric is singular at this point).

Remark 4. The reason why this procedure gives a natural metric on the 2-manifold formed by taking the connected sum of a Riemann surface of genus $\ell>0$ with $P$ is that a Riemann surface of genus $\ell>0$ with one or two boundary components always has a hyperbolic metric with constant curvature for which the boundary components are geodesics. This metric can be obtained from a pants decomposition of the Riemann surface, where each pair of pants is equipped with a hyperbolic metric for which the boundary components are geodesics. See [B].

Hence the connected sum of a Riemann surface with $P$ has a metric which is hyperbolic at all but one point, and the connected sum of a Riemann surface with two copies of $P$ has a metric which is hyperbolic at all but 2 points. To form $P$ with one disc removed, we recall that the two edges of the triangle are identified using an orientation reversing map. In Figure 1 this corresponds to identifying $\gamma_{1}$ with $\gamma_{2}$ using an orientation reversing map which maps $x_{1} \in \gamma_{1}$ to $x_{0} \in \gamma_{2}$ and maps $x_{0} \in \gamma_{1}$ to $x_{2} \in \gamma_{2}$. It turns out that the metric on the based moduli space on $P$ with one disc removed is singular at the vertex.

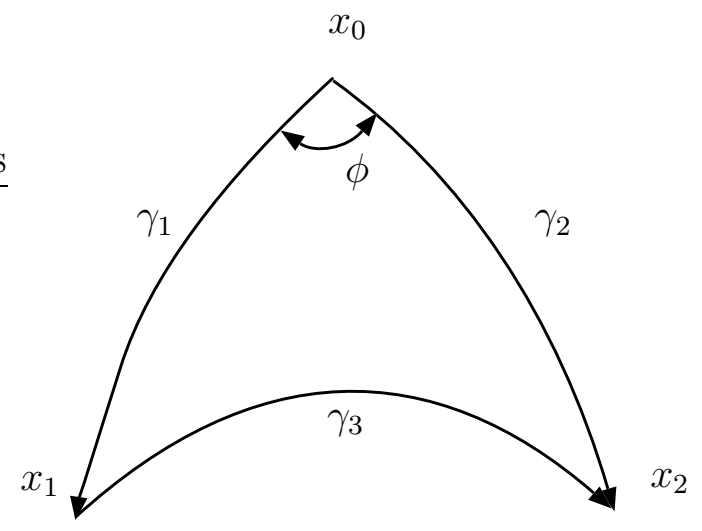

FiguRE 2. Hyperbolic geodesic triangle 
We have introduced a geodesic triangle $\triangle$ in the upper half plane (see Figure 2). As shown in Figure 2, the geodesic triangle has one distinguished vertex $x_{0}$ (which serves as the origin for geodesic polar coordinates). The sides $\gamma_{1}, \gamma_{2}, \gamma_{3}$ are geodesics. We introduce geodesic polar coordinates $(\rho, \sigma)$ (using the hyperbolic metric on the upper half plane, which is assumed to contain the triangle) with $x_{0}$ as the origin: here $\rho$ is the distance from a chosen point $x_{0}$ and $\sigma$ is the polar angle. It is assumed that the geodesic $\gamma_{1}$ is defined by the equation $\sigma=0$, and $\gamma_{2}$ is defined by $\sigma=\phi$ (where $\phi$ is the angle at $x_{0}$ ). The curves $\gamma_{1}$ and $\gamma_{2}$ have lengths $L_{1}$ and $L_{2}$ which are the same here. The geodesic $\gamma_{3}$ is specified by Coxeter's equation

$$
\operatorname{coth} \rho=\frac{\cos \left(\sigma-\sigma_{0}\right)}{\ell}, \text { see } \operatorname{Cox} \text { p.376, }
$$

where $\ell, \sigma_{0}$ are constants determined by the geodesic $\gamma_{3}$. Also,

$$
\operatorname{coth} \rho=\frac{\cosh \rho}{\sinh \rho}=\frac{\cosh ^{2}(\rho / 2)+\sinh ^{2}(\rho / 2)}{2 \sinh (\rho / 2) \cosh (\rho / 2)}=\frac{1}{2}\left(\tau+\frac{1}{\tau}\right)
$$

for $\tau=\tanh (\rho / 2)$. Thus

$$
\left(1-\frac{\cos \left(\sigma-\sigma_{0}\right)}{\ell \tau}\right) d \tau=\frac{-\sin \left(\sigma-\sigma_{0}\right)}{\ell} d \sigma .
$$

By (20), it is clear that since $\rho(\sigma=0)$ must be equal to $\rho(\sigma=\phi)$ for a hyperbolic geodesic triangle two sides of which will be identified to form $P \backslash D$, we need $\sigma_{0}=\phi / 2$ in (20).

Now the equation for $\gamma_{3}$ is (see equation (20) )

$$
\cos (\sigma-\phi / 2) \tanh \rho=\cos (\phi / 2) \tanh L
$$

and

$$
\tanh \rho=\frac{2 \tau}{1+\tau^{2}}
$$

So we have

$$
\tau\left(\gamma_{3}(\sigma)\right)=\frac{\cos (\sigma-\phi / 2)}{l}-\sqrt{\left(\frac{\cos (\sigma-\phi / 2)}{l}\right)^{2}-1}, l=\cos (\phi / 2) \tanh L
$$

Thus by (18)

$$
\begin{gathered}
\int_{\mathcal{D}} \operatorname{Tr}(d \xi \wedge * d \xi)=\frac{k \phi}{2}<Y, Y>\left[\tanh \left(\frac{L}{2}\right)\right]^{2 k} \\
-\frac{k}{2}<Y, Y>\int_{0}^{\phi}\left[\frac{\cos (\sigma-\phi / 2)}{l}-\sqrt{\left(\frac{\cos (\sigma-\phi / 2)}{l}\right)^{2}-1}\right]^{2 k} d \sigma .
\end{gathered}
$$


Let $x=\cos (\sigma-\phi / 2)$;

$$
\begin{gathered}
\int_{\mathcal{D}} \operatorname{Tr}(d \xi \wedge * d \xi)=\frac{k \phi}{2}<Y, Y>[\tanh (L / 2)]^{2 k} \\
-\frac{k}{2}<Y, Y>\left[\int_{\cos (\phi / 2)}^{1}\left(\frac{x}{l}-\sqrt{\left(\frac{x}{l}\right)^{2}-1}\right)^{2 k} \frac{d x}{\sqrt{1-x^{2}}}\right. \\
\left.-\int_{1}^{\cos (\phi / 2)}\left(\frac{x}{l}-\sqrt{\left(\frac{x}{l}\right)^{2}-1}\right)^{2 k} \frac{d x}{\sqrt{1-x^{2}}}\right] \\
=<Y, Y>\frac{k \phi}{2}[\tanh (L / 2)]^{2 k}-k<Y, Y>\int_{\cos (\phi / 2)}^{1}\left(\frac{x}{l}-\sqrt{\left(\frac{x}{l}\right)^{2}-1}\right)^{2 k} \frac{d x}{\sqrt{1-x^{2}}}
\end{gathered}
$$

Consider a more sp ecific geodesic triangle, the triangle with two sides of equal length $L$. This means $\sigma$ is fixed on those two sides and the third side is determined by Coxeter's equation (20), where $(\rho, \sigma)$ are the polar coordinates as defined above. By (14) we have

$$
\xi(\rho, \sigma)=Y(\exp u(\rho))^{k} \cos k \sigma
$$

where in the notation of (8),$u=\tanh (\rho / 2)$ in the case of a hyperbolic metric.

We recall from (17) that

$$
\begin{aligned}
& \int_{\triangle} \operatorname{Tr}(d \xi \wedge * d \xi)=\int_{C_{1}} \operatorname{Tr}(\xi \wedge * d \xi)-\int_{\mathcal{D}} \operatorname{Tr}(d \xi \wedge * d \xi) \\
& =k\left\langle Y, Y>\int_{\cos (\phi / 2)}^{1}\left(\frac{x}{l}-\sqrt{\left(\frac{x}{l}\right)^{2}-1}\right)^{2 k} \frac{d x}{\sqrt{1-x^{2}}}\right.
\end{aligned}
$$

We will compute this integral in Appendix A.

We denote the integral (23) by $k<Y, Y>h(\phi)$ because although it depends on two parameters $\phi$ and $L$, there is a relation between these two. We can see this as follows. Assume the length of $\gamma_{3}$ is fixed (since $\gamma_{3}$ will be glued to the boundary of a Riemann surface); denote this length by $b$. We have

$$
\begin{gathered}
\cosh ^{2} L=\frac{\sinh ^{2} b}{2(\cosh b-1)}+\frac{(\cosh b-1)}{2} \frac{(1+\cos \phi)^{2}}{1-\cos ^{2} \phi} \\
\frac{2(\cosh b-\cos \phi)(\cosh b-1)(\cos \phi+1)}{2(\cosh b-1)\left(1-\cos ^{2} \phi\right)} \\
=\frac{\cosh b-\cos \phi}{1-\cos \phi}
\end{gathered}
$$

This is the relation between $\phi$ and $L$ once $b$ is fixed. 
If $\tau(L)=\tanh L / 2$, then the relation between $\tau(L)$ and $\phi$ is

$$
\tau^{2}(\phi)=\tau^{2}(L(\phi))=\frac{\sqrt{\frac{\cosh b-\cos \phi}{1-\cos \phi}}-1}{\sqrt{\frac{\cosh b-\cos \phi}{1-\cos \phi}}+1} ;
$$

thus $h$ is a function of the top angle $\phi$ only.

Thus from (23) we have

$$
<d \xi, d \xi>=k<Y, Y>h(\phi)
$$

If we choose an orthonormal basis $e_{1}, e_{2}, \cdots, e_{n}$ for $\operatorname{Lie}(G)$, then the volume element of this moduli space using the metric $\Gamma$ is $\sqrt{\operatorname{det} \Gamma}$, and

$$
\operatorname{det} \Gamma=(k h(\phi))^{n}, \text { using (26) }
$$

where $n$ is the dimension of $\operatorname{Lie}(G)$ i.e. $\mathfrak{g}$.

2.3. Euclidean metric on the geodesic triangle. The only smooth constant curvature metric on $P \backslash D$ for which the boundary is a geodesic is the Euclidean metric. For the geodesic triangle $\triangle$ equipped with the Euclidean metric, we get

$$
u=\int^{\rho} \frac{d \rho}{\rho}=\ln \rho
$$

so $\tau=\rho$ and

$$
\xi=Y \rho^{k} \cos k \sigma .
$$

The geodesic $\gamma_{3}$ is given by

$$
\rho \cos (\sigma-\phi / 2)=L
$$

so we have

$$
\int_{C_{1}} \operatorname{Tr}(\xi \wedge * d \xi)=\frac{\pi}{2}<Y, Y>L^{2 k}
$$

and from (16) and (18)

$$
\begin{aligned}
& \int_{\mathcal{D}} \operatorname{Tr}(d \xi \wedge * d \xi)=\frac{\pi}{2}<Y, Y>L^{2 k}-\frac{k}{2}<Y, Y>\int_{\rho \cos (\sigma-\phi / 2)=L} \rho^{2 k} d \sigma \\
& (28) \quad=\frac{\pi}{2}<Y, Y>L^{2 k}-k<Y, Y>L^{2 k} \int_{0}^{\phi / 2} \sec ^{2 k}(\sigma) d \sigma .
\end{aligned}
$$

Thus the norm $\int_{\mathcal{D}} \operatorname{Tr}(d \xi \wedge * d \xi)$ is given by $k<Y, Y>h(\phi)$ for an appropriate function $h(\phi)$ which depends on the angle $\phi$ and on the choice of Euclidean metric. 
2.4. Spherical metric on the geodesic triangle. For a spherical metric on the geodesic triangle, we get

$$
u=\int^{\rho} \frac{d x}{\sin x}=\ln \tan (\rho / 2)
$$

so $\tau=\tan (\rho / 2)$. We then get that

$$
\int_{C_{1}} \operatorname{Tr}(\xi \wedge * d \xi)=\frac{\pi}{2}<Y, Y>(\tan (L / 2))^{2 k}
$$

Hence we get that

$$
\int_{\mathcal{D}} \operatorname{Tr}(d \xi \wedge * d \xi)=<Y, Y>\left\{\frac{\pi}{2}(\tan L / 2)^{2 k}-\frac{k}{2} \int_{\gamma_{3}}(\tan \rho / 2)^{2 k} d \sigma\right\}
$$

Here, $\gamma_{3}$ is the segment of the great circle connecting the points $x_{1}=$ $(\rho=L, \sigma=0)$ and $x_{2}=(\rho=L, \sigma=\phi)$. The length of this geodesic and the angles at the vertices $x_{1}, x_{2}$ can be determined using spherical trigonometry Weis. Thus when $\triangle$ is equipped with a spherical metric of constant curvature, the norm $<d \xi, d \xi>$ is given by $k<Y, Y>h(\phi)$ for an appropriate function $h(\phi)$ which depends on $\phi$ and on the choice of spherical metric.

2.5. $\Sigma$ is the connected sum of a Riemann surface with $P$. To consider the volume of the moduli space of the connected sum of a Riemann surface with $P$, first, let us examine the following lemmas:

Lemma 5. Let $E$ be a Riemannian manifold with metric $\Gamma$. Let $f$ : $E \rightarrow \mathbb{R}$ be a smooth function for which $d f_{m} \neq 0$ for any $m \in E$. Then

$$
\operatorname{Vol}(E)=\int_{\mathbb{R}} \frac{\operatorname{Volf} f^{-1}(t)}{d f(v(t))} d t
$$

where $v(t)$ is the unit normal vector to $f^{-1}(t)$.

Proof. The volume form on $T_{m} E$ is $e_{1}^{*} \wedge \cdots \wedge e_{n}^{*}$ where $\left\{e_{j}\right\}$ is an orthonormal basis of tangent vectors i.e. $\Gamma\left(e_{j}, e_{k}\right)=\delta_{j k}$ and $e_{i}^{*}$ are the dual basis vectors for $T_{m}^{*} E$ for any $m \in E$. Choose $e_{1}, \cdots, e_{n-1} \in$ $T_{m}\left(f^{-1}(t)\right)$ so $e_{1}^{*} \wedge \cdots \wedge e_{n-1}^{*}$ is the volume form on $T_{m}\left(f^{-1}(t)\right)$. Then 
$e_{n}^{*}=\frac{d f}{d f(v(t))}$ since $v(t)$ is the unit vector normal to $f^{-1}(t)$. Thus

$$
\begin{aligned}
\operatorname{Vol}(E) & =\int_{E}\left(e_{1}^{*} \wedge \cdots \wedge e_{n-1}^{*}\right) \wedge e_{n}^{*} \\
& =\int_{E}\left(e_{1}^{*} \wedge \cdots \wedge e_{n-1}^{*}\right) \wedge \frac{d f}{d f(v(t))} \\
& =\int_{t \in \mathbb{R}} d t \int_{f^{-1}(t)} \frac{(d v o l)}{d f(v(t))} \\
& =\int_{\mathbb{R}} \frac{\operatorname{Vol}\left(f^{-1}(t)\right)}{d f(v(t))} d t
\end{aligned}
$$

Similarly, we have the following lemma,

Lemma 6. Let $E$ be a Riemannian manifold with metric $\Gamma$, and suppose $f: E \rightarrow G$ is a smooth map for which df has maximal rank at generic points in $E$. Then

$$
\operatorname{Vol}(E)=\int_{g \in G} \frac{\operatorname{Vol}\left(f^{-1}(g)\right)}{d g\left(\wedge_{j=1}^{N} d f\left(v_{j}\right)\right)} d g
$$

where $d g$ is the volume element given by a Riemannian metric on $G$ and $v_{1}, \cdots, v_{N}$ are unit normal vectors to $f^{-1}(g)$ with $N=\operatorname{dim} G$.

Now let us return to our particular situation. ${ }^{1}$ We have a nonorientable surface $\Sigma$ which is the connected sum of a Riemann surface $\Sigma_{1}$ with a nonorientable surface $\Sigma_{2}$ (either one or two copies of the projective plane $P)$. Denote $E_{1}=\operatorname{Hom}\left(\pi_{1}\left(\Sigma_{1}\right), G\right)$ where $\Sigma_{1}$ is a Riemann surface of genus $\ell$ with one boundary component, and $E_{2}=$ $\operatorname{Hom}\left(\pi_{1}\left(\Sigma_{2}\right), G\right)$ where in this section $\Sigma_{2}=P$ with one disc removed. Here $\pi_{1}\left(\Sigma_{2}\right)=\left\{x, y \mid x=y^{2}\right\} \cong Z$ so $\operatorname{Hom}\left(\pi_{1}\left(\Sigma_{2}\right), G\right) \cong G$. Define maps $f_{i}: E_{i} \rightarrow G$ for $i=1,2$ by sending a representation to its value on the loop around the boundary. Let

$$
E=\left\{\left(m_{1}, m_{2}\right) \in E_{1} \times E_{2} \mid f_{1}\left(m_{1}\right)=f_{2}\left(m_{2}\right)\right\}
$$

Now we use Lemma 6 with $E_{2} \cong G$ and let $f: E \rightarrow G=E_{2}$ be the map $\left(m_{1}, m_{2}\right) \mapsto m_{2}$. Notice that in this case the hypothesis of $f$ having maximal rank is valid generically because this reduces to $d f_{1}: T E_{1} \rightarrow \mathfrak{g}$ being surjective, and it was proved by Goldman Go1] (Proposition 3.7) that the image of $d f_{1}$ at a point $A=\left(a_{1}, \ldots, a_{\ell}, b_{1}, \ldots, b_{\ell}\right) \in E_{1}=G^{2 \ell}$ is $z(A)^{\perp}$ (the orthocomplement of the Lie algebra of the stabilizer of $A$ under the adjoint action), and $z(A)=0$ for generic $A$.

\footnotetext{
${ }^{1}$ For other approaches to measures on moduli spaces, see [Fine, [Fo, [Liu and BeSe, Se1, Se2, Se3].
} 
We have

$$
\operatorname{Vol}(E)=\int_{G} \frac{\operatorname{Vol}\left(f^{-1}(g)\right)}{d g\left(\wedge_{j} d f\left(v_{j}\right)\right)} d g .
$$

Note that the metric on $E$ is $\pi_{1}^{*} h_{1}+\pi_{2}^{*} h_{2}$ where $h_{1}$ is the metric on $E_{1}$ and $h_{2}$ the metric on $E_{2}$ which are given by the equation (26) i.e. (Haar measure) $\times(k h(\phi))^{n / 2}$. Thus we can choose an orthonormal basis $\left\{v_{j}\right\}$ in $\mathfrak{g}$ and $d g\left(\wedge_{j} d f\left(v_{j}\right)\right)=1$. Thus we have $\operatorname{Vol}\left(f^{-1}(g)\right)=$ $\operatorname{Vol}\left(f_{1}^{-1}\left(g^{2}\right)\right) \operatorname{Vol}\left(f_{2}^{-1}\left(g^{2}\right)\right)$ because $f^{-1}(g)=f_{1}^{-1}\left(g^{2}\right) \times f_{2}^{-1}\left(g^{2}\right)$. It follows that

$$
\operatorname{Vol}(E)=\int_{g \in G} \operatorname{Vol}\left(f_{1}^{-1}\left(g^{2}\right)\right) \operatorname{Vol}\left(f_{2}^{-1}\left(g^{2}\right)\right) d g
$$

where our moduli space is $\mathcal{M}=E / G$. Thus we have $\operatorname{Vol}(E)=$ $\operatorname{Vol}(\mathcal{M}) \operatorname{Vol}(G)$.

Notice that the moduli space of gauge equivalence classes of flat connections on a Riemann surface with one boundary component about which the holonomy is constrained to take a fixed value (in other words the moduli space of parabolic bundles) is a Kähler manifold (see for instance [AB]) so its symplectic volume (as specified by Witten's formula) is equal to its Riemannian volume (for a metric derived from any metric on the Riemann surface). Hence our procedure will give the Riemannian volume on the moduli space of gauge equivalence classes of flat connections on the connected sum.

We know from [W] the volume formula [W] (4.114) for a moduli space of flat connections on a compact orientable surface $\Sigma_{\ell}$ of genus $\ell$ with one boundary component: ${ }^{2}$ For $s \in G$, we define

$$
\begin{gathered}
\mathcal{M}\left(\Sigma_{\ell}, s\right)=\left\{\left(a_{1}, \ldots, a_{2 \ell}\right) \in G^{2 \ell} \mid \prod_{j=1}^{\ell} a_{2 j-1} a_{2 j} a_{2 j-1}^{-1} a_{2 j}^{-1} \in C(s)\right\} / G \\
=\left\{\rho \in \operatorname{Hom}\left(\pi_{1}\left(\Sigma_{\ell}-D\right), G\right) \mid \rho\left(\left[\partial\left(\Sigma_{\ell}-D\right)\right]\right) \in C(s)\right\} / G
\end{gathered}
$$

where $C(s)$ is the conjugacy class of $s$. We also define

$$
\begin{aligned}
& \mathcal{R}\left(\Sigma_{\ell}, s\right)=\left\{\left(a_{1}, \ldots, a_{2 \ell}\right) \in G^{2 \ell} \mid \prod_{j=1}^{\ell} a_{2 j-1} a_{2 j} a_{2 j-1}^{-1} a_{2 j}^{-1}=s\right\} \\
& =\left\{\rho \in \operatorname{Hom}\left(\pi_{1}\left(\Sigma_{\ell}-D\right), G\right) \mid \rho\left(\left[\partial\left(\Sigma_{\ell}-D\right)\right]\right)=s\right\}
\end{aligned}
$$

so that

$$
\mathcal{M}\left(\Sigma_{\ell}, s\right)=G \mathcal{R}\left(\Sigma_{\ell}, s\right) / G
$$

\footnotetext{
${ }^{2}$ In Section 3.1 we give a new proof of this formula.
} 
It follows that

$$
\operatorname{Vol} \mathcal{M}\left(\Sigma_{\ell}, s\right)=\operatorname{Vol} \mathcal{R}\left(\Sigma_{\ell}, s\right) \frac{\operatorname{Vol} C(s)}{\operatorname{Vol} G}
$$

If $s \in G$, then Witten's formula reads

$$
\operatorname{Vol}\left(\mathcal{M}\left(\Sigma_{\ell}, s\right)\right)=C_{1} \sum_{\alpha} \frac{1}{(\operatorname{dim} \alpha)^{2 \ell-1}} \chi_{\alpha}(s) \sqrt{F(s)},
$$

where $\alpha$ runs over all isomorphism classes of irreducible representations of $G$, the representation $\alpha$ has character $\chi_{\alpha}$, and the constant $C_{1}$ is

$$
C_{1}=\frac{\sharp Z(G) \operatorname{Vol}(G)^{2 \ell-2+1}}{(2 \pi)^{\operatorname{dim} \mathcal{M}\left(\Sigma_{\ell}, s\right)} \operatorname{Vol}(T)}
$$

and

$$
\operatorname{dim} \mathcal{M}\left(\Sigma_{\ell}, s\right)=(2 \ell-2)|G|+|G|-|T|,
$$

where $|G|$ denotes the dimension of $G$ and $|T|$ denotes the dimension of the maximal torus $T$. Note also that $F(s)$ is the Riemannian volume of the conjugacy class $C(s)$ through $s$ as defined in $[\mathrm{W}](4.53)$

$$
v=\frac{\operatorname{Vol}(G)}{\operatorname{Vol}(T)} v_{0} F(s) \text {. }
$$

(See Chapter 7, $[\mathrm{BGV}]$.) The Liouville volume of $C(s)$ is

$$
\sqrt{F(s)} \operatorname{vol} G / \operatorname{vol} T
$$

(see [AMW, Proposition 3.6.) Here $v$ represents the measure on $T / W$ obtained by pushing down the Haar measure on $G$ (under the natural map from a group element to its conjugacy class) and $v_{0}$ represents the measure on $T / W$ obtained by restricting the metric on $\mathfrak{g}$ to $\operatorname{Lie}(T)$ and then identifying $\operatorname{Lie}(T)$ with the tangent space to $T / W$. A more detailed explanation of $F(s)$ is given in the next section.

So (34) is equivalent to

$$
\operatorname{VolM}\left(\Sigma_{\ell}, s\right)=\operatorname{Vol} \mathcal{R}\left(\Sigma_{\ell}, s\right) \frac{\sqrt{F(s)}}{\operatorname{VolT}}
$$

and (35) is equivalent to

$$
\operatorname{Vol} \mathcal{R}\left(\Sigma_{\ell}, s\right)=C_{1} \operatorname{VolT} \sum_{\alpha} \frac{1}{(\operatorname{dim} \alpha)^{2 \ell-1}} \chi_{\alpha}(s) .
$$

Thus the volume of the moduli space of flat connections on the connected sum of a Riemann surface with a projective plane is

$$
\operatorname{Vol}(\mathcal{R}(\Sigma))=\operatorname{Vol}\left(\mathcal{R}\left(\Sigma_{\ell} \sharp P\right)\right)
$$




$$
=\int_{s \in G} \operatorname{Vol}\left(\mathcal{R}\left(\Sigma_{\ell}, s\right)\right) \operatorname{Vol}(\mathcal{R}(P, s)) d s
$$

Denote by $R$ the map $G \rightarrow G$,

$$
R: g \mapsto g^{2}
$$

Weyl's integral formula

$$
|W| \int_{G} f(g) d g=\int_{T}\left[F(t) \int_{G} f\left(g t g^{-1}\right) d g\right] d t
$$

gives us that

$$
\int_{G} f(g) d g=\int_{T / W} F(s) f(s) \frac{\operatorname{Vol}(G)}{\operatorname{Vol}(T)} d s .
$$

when $f$ is conjugation invariant.

Thus

$$
\begin{aligned}
\operatorname{Vol}(\mathcal{R}(\Sigma)) & =\int_{G} C_{1} \operatorname{VolT} \sum_{\alpha} \frac{1}{(\operatorname{dim}(\alpha))^{2 \ell-1}} \chi_{\alpha}(g) R_{*}(\sqrt{\operatorname{det} \Gamma} d g) \\
& =C_{1} \operatorname{Vol}(T) \sum_{\alpha} \frac{1}{(\operatorname{dim}(\alpha))^{2 \ell-1}} \int_{G} \chi_{\alpha}\left(g^{2}\right) \sqrt{\operatorname{det} \Gamma} d g
\end{aligned}
$$

The above is true because $S^{2}-D-D$ is the double cover of $P \backslash D$; the space of flat connections on $S^{2}-D-D$ (modulo based gauge transformations) is isomorphic to $G$, so we pull back the integral to $G$ under the covering map. We get

$$
\operatorname{Vol}(\mathcal{R}(\Sigma))=C_{1} \operatorname{VolT} \sum_{\alpha} \frac{1}{(\operatorname{dim}(\alpha))^{2 \ell-1}} f_{\alpha} \operatorname{Vol}(G)(k h(\phi))^{|G| / 2}
$$

by using

$$
\int_{G} \chi_{\alpha}\left(g^{2}\right) d g=f_{\alpha} \operatorname{Vol}(G)
$$

(cf: $[\mathrm{W}](2.70))$ where $f_{\alpha}=1,-1,0$ depending on whether the representation $\alpha$ admits a symmetric invariant bilinear form, an antisymmetric bilinear form, or no invariant bilinear form at all. Here $\operatorname{dim}(\alpha)$ is the dimension of the representation $\alpha$, and its character is denoted by $\chi_{\alpha}$.

We get

$$
\operatorname{Vol}(\mathcal{R}(\Sigma))=\frac{\sharp Z(G) \operatorname{Vol}(G)^{2 \ell}}{(2 \pi)^{(2 \ell-1)|G|-|T|}} H(\phi) \sum_{\alpha} \frac{f_{\alpha}}{(\operatorname{dim}(\alpha))^{2 \ell-1}}
$$

where $H(\phi)=(k h(\phi))^{|G| / 2}$ and $k=\pi / \phi$. Here $h$ is a real-valued function of the angle $\phi$ which depends on the choice of metric on the Möbius strip. 
We compare our result to Witten's formula W] (4.93)

$$
\operatorname{Vol}(\mathcal{R}(\Sigma))=\frac{\sharp Z(G) \operatorname{Vol}(G)^{2 \ell}}{(2 \pi)^{(2 \ell-1)|G|}} \sum_{\alpha} \frac{f_{\alpha}}{(\operatorname{dim}(\alpha))^{2 \ell-1}}
$$

Our formula differs from Witten's by a multiplicative factor of $H(\phi)$ $(2 \pi)^{|T|}$. The factor $(2 \pi)^{|T|}$ is due to Witten's choice of a different normalization. The factor $H(\phi)$ results from our choice of a metric on the nonorientable part of the surface (the projective plane). Note Witten did not choose a metric on $P$. On the other hand our formula for $\operatorname{Vol}(\mathcal{M})$ is a function of the angle $\phi$ for a fixed surface.

2.6. $\Sigma$ is a connected sum of a Riemann surface with two projective planes. We know from [W] the volume formula for a moduli space of flat connections on a compact orientable surface of genus $\ell$ with two boundary components [W] (4.114) (where $s_{1}, s_{2} \in G$ and the holonomies around the two boundary components are fixed at $s_{1}$ and $\left.s_{2}\right)$ : in this case Witten's formula [W] (4.114) reads as follows.

$$
\operatorname{Vol}\left(\mathcal{M}\left(\Sigma_{\ell}, s_{1}, s_{2}\right)\right)=C_{2} \sum_{\alpha} \frac{1}{(\operatorname{dim}(\alpha))^{2 \ell}} \chi_{\alpha}\left(s_{1}\right) \sqrt{F\left(s_{1}\right)} \chi_{\alpha}\left(s_{2}\right) \sqrt{F\left(s_{2}\right)},
$$

where the constant $C_{2}$ is

$$
\frac{\sharp Z(G) \operatorname{Vol}(G)^{2 \ell}}{(2 \pi)^{\operatorname{dim} \mathcal{M}\left(\Sigma_{\ell}, s_{1}, s_{2}\right)} \operatorname{Vol}(T)^{2}},
$$

and

$$
\operatorname{dim} \mathcal{M}\left(\Sigma_{\ell}, s_{1}, s_{2}\right)=(2 \ell-2)|G|+2|G|-2|T| .
$$

Again we define

$\mathcal{R}\left(\Sigma_{\ell}, s_{1}, s_{2}\right)=\left\{\rho \in \operatorname{Hom}\left(\pi_{1}\left(\Sigma_{\ell}-D_{1}-D_{2}\right), G\right) \mid \rho\left(\left[\partial D_{1}\right]=s_{1}, \rho\left(\left[\partial D_{2}\right]=s_{2}\right\}\right.\right.$.

Here we have chosen representatives $\left[\partial D_{j}\right]$ for the elements of the fundamental group represented by the loops around the $j$-th boundary component, by connecting the basepoint to some point on the boundary. Thus

$$
\mathcal{M}\left(\Sigma_{\ell}, s_{1}, s_{2}\right)=(G \times G) \mathcal{R}\left(\Sigma_{\ell}, s_{1}, s_{2}\right) / G \times G
$$

where $\left(g_{1}, g_{2}\right) \in G \times G$ acts on $\operatorname{Hom}\left(\pi_{1}\left(\Sigma_{\ell}-D_{1}-D_{2}\right), G\right)$ so that if the value of the representation of the loop around the $j$-th boundary component is $s_{j}$, then this value becomes $g_{j} s_{j} g_{j}^{-1}$. Following (34) and (37) above, we have

$$
\operatorname{VolM}\left(\Sigma_{\ell}, s_{1}, s_{2}\right)=\operatorname{Vol} \mathcal{R}\left(\Sigma_{\ell}, s_{1}, s_{2}\right) \frac{\operatorname{VolC}\left(s_{1}\right) \operatorname{VolC}\left(s_{2}\right)}{(\operatorname{Vol} G)^{2}}
$$




$$
=\operatorname{Vol} \mathcal{R}\left(\Sigma_{\ell}, s_{1}, s_{2}\right) \frac{\sqrt{F\left(s_{1}\right)} \sqrt{F\left(s_{2}\right)}}{(\operatorname{VolT})^{2}} .
$$

Equivalently, Witten's formula is

$$
\operatorname{Vol}\left(\mathcal{R}\left(\Sigma_{\ell}, s_{1}, s_{2}\right)\right)=C_{2}(\operatorname{Vol}(T))^{2} \sum_{\alpha} \frac{1}{(\operatorname{dim}(\alpha))^{2 \ell}} \chi_{\alpha}\left(s_{1}\right) \chi_{\alpha}\left(s_{2}\right)
$$

Thus the volume of the moduli space of flat connections on the connected sum of a Riemann surface with two projective planes (equivalently, the connected sum with one Klein bottle) is

$$
\begin{aligned}
& \operatorname{Vol}(\mathcal{R}(\Sigma))=\operatorname{Vol}\left(\mathcal{R}\left(\Sigma_{\ell} \sharp P \sharp P\right)\right) \\
& =\int_{G \times G} d s_{1} d s_{2} \operatorname{Vol}\left(\mathcal{R}\left(\Sigma_{\ell}, s_{1}, s_{2}\right)\right) \operatorname{Vol}\left(\mathcal{R}\left(P, s_{1}\right)\right) \operatorname{Vol}\left(\mathcal{R}\left(P, s_{2}\right)\right)
\end{aligned}
$$

Let $\sqrt{\operatorname{det} \Gamma_{1}}$ and $\sqrt{\operatorname{det} \Gamma_{2}}$ denote the volume elements on the two copies of $P \backslash D$ respectively. We have

$$
\begin{aligned}
& \operatorname{Vol}(\mathcal{R}(\Sigma))=\int_{G \times G} \operatorname{Vol}\left(\mathcal{R}\left(\Sigma_{\ell}, g_{1}, g_{2}\right)\right) R_{*}\left(\sqrt{\operatorname{det} \Gamma_{1}} d g_{1}\right) R_{*}\left(\sqrt{\operatorname{det} \Gamma_{2}} d g_{2}\right) \\
& =C_{2}(V o l T)^{2} \sum_{\alpha} \frac{1}{(\operatorname{dim}(\alpha))^{2 \ell}} \int_{G \times G} \chi_{\alpha}\left(g_{1}\right) \chi_{\alpha}\left(g_{2}\right) R_{*}\left(\sqrt{\operatorname{det} \Gamma_{1}} d g_{1}\right) R_{*}\left(\sqrt{\operatorname{det} \Gamma_{2}} d g_{2}\right) \\
& =C_{2}(V o l T)^{2} \sum_{\alpha} \frac{1}{(\operatorname{dim}(\alpha))^{2 \ell}} \sqrt{\operatorname{det} \Gamma_{1}} \sqrt{\operatorname{det} \Gamma_{2}}\left(\int_{G} \chi_{\alpha}\left(g^{2}\right) d g\right)^{2}
\end{aligned}
$$

where $R$ was defined by (41). We use equation (42) and thus the volume becomes

$$
\operatorname{Vol}(\mathcal{R}(\Sigma))=\frac{\sharp Z(G) \operatorname{Vol}(G)^{2 \ell-1}}{(2 \pi)^{(2 \ell)|G|-2|T|}} \sum_{\alpha} \frac{1}{(\operatorname{dim}(\alpha))^{2 l}} f_{\alpha}^{2} H\left(\phi_{1}\right) H\left(\phi_{2}\right) \operatorname{Vol}(G)^{2}
$$

where $H\left(\phi_{i}\right)=\left(k_{i} h\left(\phi_{i}\right)\right)^{|G| / 2}$, the angles $\phi_{1}$ and $\phi_{2}$ are the top angles of the two triangles respectively, $k_{i}=\pi / \phi_{i}$, and $f_{\alpha}$ is defined as in Section 2.5. Note that $f_{\alpha}^{2}=1$ if $\alpha=\bar{\alpha}$ and $f_{\alpha}^{2}=0$ otherwise. We get the final formula

$$
\operatorname{Vol}(\mathcal{R}(\Sigma))=\frac{\sharp Z(G) \operatorname{Vol}(G)^{2 \ell+1}}{(2 \pi)^{(2 \ell)|G|-2|T|}} H\left(\phi_{1}\right) H\left(\phi_{2}\right) \sum_{\alpha=\bar{\alpha}} \frac{1}{(\operatorname{dim}(\alpha))^{2 \ell}} .
$$

We compare this with Witten's formula W] (4.77)

$$
\operatorname{Vol}(\mathcal{R}(\Sigma))=\frac{\sharp Z(G) \operatorname{Vol}(G)^{2 \ell+1}}{(2 \pi)^{(2 \ell)|G|}} \sum_{\alpha=\bar{\alpha}} \frac{1}{(\operatorname{dim}(\alpha))^{2 \ell}}
$$


Our formula differs from Witten's formula by a multiplicative factor of $H\left(\phi_{1}\right) H\left(\phi_{2}\right)(2 \pi)^{2(|T|)}$. The factor $(2 \pi)^{2(|T|)}$ is due to Witten's choice of a different normalization. The factors $H\left(\phi_{i}\right)$ result from our choice of a metric on the two projective planes.

Notice that there are two factors $H\left(\phi_{i}\right)$ (in contrast to the case when the manifold is a connected sum of a Riemann surface with one copy of $P$, when there is only one such factor). Thus $\operatorname{Vol}(\mathcal{M})$ is a function of the angles $\phi_{1}, \phi_{2}$ for a fixed surface $\Sigma_{l}$.

We may summarize our conclusions as follows. When we take the connected sum of a Riemann surface with a real projective plane formed by identifying two sides of length $L$ of a geodesic triangle with polar angle $\phi$, the volume we have obtained is a function of the angle $\phi$ since the length $L$ of the side of the triangle is determined once the length of the third edge of the triangle is given. A pair $(L, \phi)$ gives a conformal structure on the triangle. Once we fix a conformal structure, we obtain a volume for the moduli space determined by this structure. The situation is similar for the connected sum of a Riemann surface with two projective planes.

\section{WitTen's vOlume FORMUla AND HaAR MEASURE}

In this section, we will consider Witten's volume formula for the moduli space of flat connections on a 2-manifold, and give a new proof of these formulas using Haar measure. Notice that Witten did not give a proof exclusively using Haar measure to compute the volume of the moduli space. Witten gave three arguments for the formula in [W]:

- In Section 2, Witten used results of Migdal [Mi] involving lattice gauge theories to compute the Yang-Mills partition function in two dimensions. Migdal's result only makes sense when the Yang-Mills partition function has been regularized, giving a result which reduces to the volume of the moduli space when the regularization parameter tends to 0 .

- In Section 3, Witten deduced the result from the Verlinde formula, which is a formula for the Riemann-Roch number of the prequantum line bundle of the moduli space $M$ of flat connections on a 2-manifold, $R R\left(M, L^{k}\right)$. Since the cohomology class of the symplectic form $\omega$ is the first Chern class of $L$, we may expect that the leading order term in the Riemann-Roch number (as a polynomial in $k$ ) is $k^{\operatorname{dim} M / 2} \operatorname{Vol}(M)$.

- In Section 4, Witten characterized the volume of the moduli space using Reidemeister-Ray-Singer torsion. He used the fact that Reidemeister torsion is multiplicative: If $M$ is the union 
of two submanifolds $M_{1}$ and $M_{2}$ with boundary $N$, glued along the boundary, then

$$
\tau(M)=\tau\left(M_{1}\right) \tau\left(M_{2}\right) / \tau(N)
$$

where the torsion is viewed as a ratio of determinants of elliptic operators and the above quotient makes sense in terms of the Mayer-Vietoris sequence which computes the cohomology groups of $M$ from those of $M_{1}, M_{2}$ and $N$.

Since the moduli space can be identified with the space of representations of a surface group (in other words $\operatorname{Hom}\left(\pi_{1}(\Sigma), G\right) / G$ ) and a compact Lie group has a natural Riemannian measure, the Haar measure, we can try to understand Witten's formula for the moduli space by pushing forward the Haar measure on $G .^{3}$

We will use the following two facts to show our argument.

- Weyl's integral formula (cf. [BD] (4.1.11))

$$
|W| \int_{G} f(g) d g=\int_{T}\left[F(t) \int_{G} f\left(g t g^{-1}\right) d g\right] d t
$$

If $f$ is conjugation invariant, the formula becomes

$$
\begin{aligned}
|W| \int_{G} f(g) d g & =\int_{T} F(t) f(t) \frac{\operatorname{Vol}(G)}{\operatorname{Vol}(T)} d t \\
& =|W| \int_{T / W} i_{*}(F(t)) f\left(i^{-1}(t)\right) \frac{\operatorname{Vol}(G)}{\operatorname{Vol}(T)} i_{*} d t \\
& =|W| \int_{s \in T / W} F\left(i^{-1}(s)\right) f\left(i^{-1}(s)\right) \frac{\operatorname{Vol}(G)}{\operatorname{Vol}(T)} d s
\end{aligned}
$$

Here $i: T \rightarrow T / W$ is the local isomorphism with $i_{*} d t=: d s$.

Thus we have

$$
\int_{G} f(g) d g=\int_{s \in T / W} F(s) f(s) \frac{\operatorname{Vol}(G)}{\operatorname{Vol}(T)} d s
$$

Recall that $F(s)=F\left(i^{-1}(s)\right)$ is the volume of the conjugacy class containing $s$ as defined for example in $[\mathrm{W}](4.53)$ :

$$
v=\frac{\operatorname{Vol}(G)}{\operatorname{Vol}(T)} v_{0} F(s),
$$

where $v$ represents the measure on $T / W$ obtained by pushing down the Haar measure on $G$ (under the natural map from a group element to its conjugacy class) and $v_{0}$ represents the measure on $T / W$ obtained by restricting the metric on $\mathfrak{g}$ to $\operatorname{Lie}(T)$ (which determines a Haar measure

\footnotetext{
${ }^{3}$ For a related treatment of the role of Haar measure in determining volumes, see AMM and $\mathrm{AMW}$.
} 
on $T$ ) and then identifying $\operatorname{Lie}(T)$ with the tangent space to $T / W$. This is as in the picture shown below:

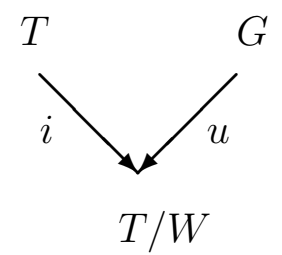

with $v=u_{*} d g$, and $v_{0}=i_{*} d t$, where $d g$ and $d t$ are the Haar measures on $G$ and $T$ respectively. Here $i: T \rightarrow T / W$ is the local isomorphism and $u: G \rightarrow T / W$ is the map which maps $g$ to its conjugacy class.

Explicitly we have

$$
F(s)=\operatorname{det}_{\mathbb{R}}(1-\operatorname{Ad}(s))
$$

or for $s=\exp \lambda$ for $\lambda \in \operatorname{Lie}(T)$ such that $\alpha(\lambda) \neq 0$ for any root $\alpha$, we have

$$
F(\exp \lambda)=\prod_{\alpha>0} 4 \sin ^{2} \alpha(\lambda)
$$

where we assume $s \in T$ and view $\operatorname{Ad}(s)$ as an endomorphism of the orthocomplement of the Lie algebra of $T$.

- Pushforward of volume under a surjective map: Suppose $p: M \rightarrow$ $N$ is a surjective map, $f: N \rightarrow R$ is a map, and $V_{M}$ and $V_{N}$ are volume forms for $M$ and $N$ respectively. Then a function $h$ characterizing the pushforward is defined by

$$
\int_{M} f(p(x)) V_{M}=\int_{N} f(y) h(y) V_{N}, \text { where } p_{*}\left(V_{M}\right)(y)=h(y) V_{N} .
$$

Roughly speaking, $h(y)=\operatorname{Vol}\left(p^{-1}(y)\right)$.

3.1. $\Sigma$ is a one punctured Riemann surface of genus $\ell+1$. The following picture shows the relations between the pushforwards. 


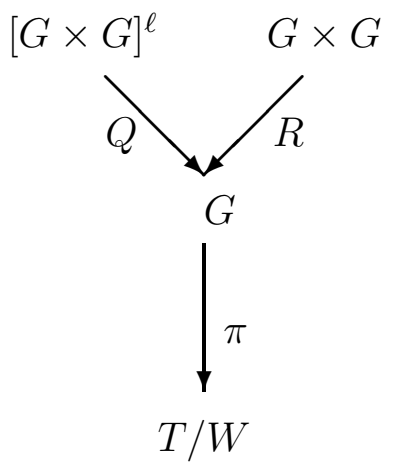

where $Q\left(A_{1}, \cdots, A_{\ell}, B_{1}, \cdots, B_{\ell}\right)=\prod_{i=1}^{\ell} A_{i} B_{i} A_{i}^{-1} B_{i}^{-1}$

$$
\text { and } R\left(g_{1}, g_{2}\right)=h g_{1} g_{2} g_{1}^{-1} g_{2}^{-1}
$$

where $h \in G$ is the holonomy around the boundary.

The volume formula for a moduli space of flat connections on a compact orientable surface with one boundary component $[\mathrm{W}](4.114)$ is as follows. For an element $s \in G$

$$
\operatorname{Vol}\left(\mathcal{R}\left(\Sigma_{\ell}, s\right)\right)=\frac{\sharp Z(G) \operatorname{Vol}(G)^{2 \ell-1}}{(2 \pi)^{(2 \ell-1)|G|-|T|}} \sum_{\alpha} \frac{1}{(\operatorname{dim}(\alpha))^{2 \ell-1}} \chi_{\alpha}(s)
$$

We want to show that Witten's formula W] (4.114) is the pushforward of the Haar measure on $G$ i.e. prove equation (48) by induction on $\ell$ (assuming that equation (48) is true for genus $\ell$, we prove it for genus $\ell+1)$. The induction hypothesis is valid for $\ell=0$ because by W] (2.43) we have

$$
\sum_{\alpha}(\operatorname{dim} \alpha) \chi_{\alpha}(s)=\delta(s-1)
$$

and the space $\mathcal{R}\left(\Sigma_{0}, s\right)$ is one point if $s=1$ and empty otherwise, so its volume is $\delta(s-1)$.

Consider a torus $\Sigma_{1}$ with one boundary component. If $\Sigma_{1}-D$ is glued to $\Sigma_{\ell}$ along the boundary of $D$, we obtain $\Sigma_{\ell+1}$. (See Figure 3.) 
Thus we have

$$
\begin{aligned}
& \operatorname{Vol}\left(\mathcal{R}\left(\Sigma_{\ell+1}, h\right)\right)=\int_{G} \operatorname{Vol}\left(\mathcal{R}\left(\Sigma_{\ell}, s\right)\right) \operatorname{Vol}\left(\mathcal{R}\left(\Sigma_{1}, s, h\right)\right) d s \\
= & \frac{\sharp Z(G) \operatorname{Vol}(G)^{2 \ell}}{(2 \pi)^{(2 \ell-1)|G|-|T|}} \sum_{\alpha} \frac{1}{(\operatorname{dim}(\alpha))^{2 \ell-1}} \int_{G} \chi_{\alpha}(g) R_{*}\left(d g_{1} \wedge d g_{2}\right) \\
= & \frac{\sharp Z(G) \operatorname{Vol}(G)^{2 \ell}}{(2 \pi)^{(2 \ell-1)|G|-|T|}} \sum_{\alpha} \frac{1}{(\operatorname{dim}(\alpha))^{2 \ell-1}} \int_{G \times G} \chi_{\alpha}\left(h g_{1} g_{2} g_{1}^{-1} g_{2}^{-1}\right) d g_{1} d g_{2} \\
= & \frac{\sharp Z(G) \operatorname{Vol}(G)^{2 \ell}}{(2 \pi)^{(2 \ell-1)|G|-|T|}} \sum_{\alpha} \frac{1}{(\operatorname{dim}(\alpha))^{2 \ell-1}} \int_{G} \chi_{\alpha}\left(h g_{1}\right) \chi_{\alpha}\left(g_{1}^{-1}\right) d g_{1} \frac{\operatorname{Vol}(G)}{\operatorname{dim}(\alpha)}
\end{aligned}
$$

where we have used ${ }^{4}$ (cf: W] $\left.(2.50)\right)$

$$
\int_{G} \chi_{\alpha}\left(A u B u^{-1}\right) d u=\frac{\operatorname{Vol}(G)}{\operatorname{dim}(\alpha)} \chi_{\alpha}(A) \chi_{\alpha}(B)
$$

and $R$ was defined by (47).

Now we use (cf:[W] (2.48))

$$
\int_{G} \chi_{\alpha}(h g) \chi_{\alpha}\left(g^{-1}\right) d g=\frac{\operatorname{Vol}(G)}{\operatorname{dim}(\alpha)} \chi_{\alpha}(h)
$$

to get our final formula for $h \in G$

$$
\operatorname{Vol}\left(\mathcal{R}\left(\Sigma_{\ell+1}, h\right)\right)=\frac{\sharp Z(G) \operatorname{Vol}(G)^{2 \ell+1}}{(2 \pi)^{(2 \ell+1)|G|-|T|}} \sum_{\alpha} \frac{\chi_{\alpha}(h)}{(\operatorname{dim}(\alpha))^{2 \ell+1}}
$$

We note that the final formula (53) is invariant under conjugation: $\operatorname{Vol}\left(\mathcal{M}\left(\Sigma_{\ell+1}, h\right)=\operatorname{Vol}\left(\mathcal{M}\left(\Sigma_{\ell+1}, k h k^{-1}\right)\right.\right.$ for any $k \in G$. So this formula only depends on the conjugacy class of $h$.

Note that Witten [W] (4.114) gives the following formula for the volume for genus $\ell+1$ with $h \in G$,

$$
\operatorname{Vol}\left(\mathcal{R}\left(\Sigma_{\ell+1}, h\right)\right)=\frac{\sharp Z(G) \operatorname{Vol}(G)^{2 \ell+1}}{(2 \pi)^{(2 \ell+1)|G|-|T|}} \sum_{\alpha} \frac{\chi_{\alpha}(h)}{(\operatorname{dim}(\alpha))^{2 \ell+1}}
$$

This agrees with our result.

Our calculation shows us that Witten's formula (4.114) can be understood in terms of the pushforward of Haar measure on $G$.

\footnotetext{
${ }^{4}$ The equations $\mathrm{W}$ (2.43), (2.48), (2.50), (2.70) and (4.50) are standard facts from the representation theory of compact Lie groups, and follow from the orthogonality relations. See also $[\mathrm{BD}$.
} 
3.2. $\Sigma$ is the connected sum of a Riemann surface of genus $\ell$ with $P$. We know from $[\mathrm{W}$ the volume formula for a moduli space of flat connections on a compact orientable surface with one boundary component $[\mathrm{W}]$ (4.114), where $s \in G$ and the holonomy around the boundary is constrained to be $s$ :

$$
\operatorname{Vol}\left(\mathcal{R}\left(\Sigma_{\ell}, s\right)\right)=C_{1} \operatorname{VolT} \sum_{\alpha} \frac{1}{(\operatorname{dim}(\alpha))^{2 \ell-1}} \chi_{\alpha}(s)
$$

where the constant $C_{1}$ is given by equation (36).

The following picture shows the relations between the pushforwards.

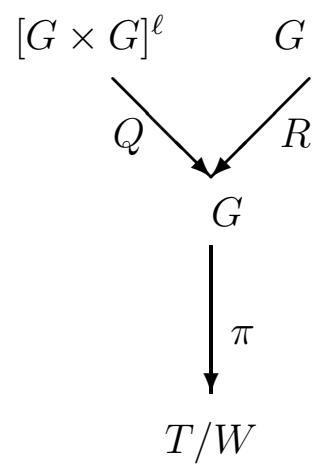

where

$$
Q\left(A_{1}, \cdots, A_{\ell}, B_{1}, \cdots, B_{\ell}\right)=\prod_{i=1}^{\ell} A_{i} B_{i} A_{i}^{-1} B_{i}^{-1}
$$

and

$$
R(g)=g^{2} .
$$

By definition of the pushforward,

$$
\operatorname{Vol}(\mathcal{R}(P, g)) d g=R_{*}(d g)
$$


Thus

$$
\begin{aligned}
\operatorname{Vol}(\mathcal{R}(\Sigma)) & =\operatorname{Vol}\left(\mathcal{R}\left(\Sigma_{\ell \sharp} P\right)\right)=\int_{s \in G} \operatorname{Vol}\left(\mathcal{R}\left(\Sigma_{\ell}, s\right)\right) \operatorname{Vol}(\mathcal{R}(P, s)) d s \\
& =\int_{G} C_{1} \operatorname{VolT} \sum_{\alpha} \frac{1}{(\operatorname{dim}(\alpha))^{2 \ell-1}} \chi_{\alpha}(g) R_{*}(d g) \\
& =C_{1} \operatorname{VolT} \sum_{\alpha} \frac{1}{(\operatorname{dim}(\alpha))^{2 \ell-1}} \int_{G} \chi_{\alpha}\left(g^{2}\right) d g \\
& =\frac{\sharp Z(G) \operatorname{Vol}(G)^{2 \ell}}{(2 \pi)^{(2 \ell-1)|G|-|T|}} \sum_{\alpha} \frac{1}{(\operatorname{dim}(\alpha))^{2 \ell-1}} f_{\alpha}
\end{aligned}
$$

where we have used equation (42) and $f_{\alpha}$ is defined in Section 2.5.

We get our final formula

$$
\operatorname{Vol}(\mathcal{R}(\Sigma))=\frac{\sharp Z(G) \operatorname{Vol}(G)^{2 \ell}}{(2 \pi)^{(2 \ell-1)|G|-|T|}} \sum_{\alpha} \frac{f_{\alpha}}{(\operatorname{dim}(\alpha))^{2 \ell-1}}
$$

Note that Witten's formula [W] (4.93) is

$$
\operatorname{Vol}(\mathcal{R}(\Sigma))=\frac{\sharp Z(G) \operatorname{Vol}(G)^{2 \ell}}{(2 \pi)^{(2 \ell-1)|G|}} \sum_{\alpha} \frac{f_{\alpha}}{(\operatorname{dim}(\alpha))^{2 \ell-1}}
$$

Our formula differs from Witten's by a multiplicative factor of $(2 \pi)^{|T|}$ because Witten chose a different normalization.

3.3. $\Sigma$ is the connected sum of a Riemann surface of genus $\ell$ with the Klein bottle. Again, the following picture shows the relations between the pushforwards. (Note that we will have a similar calculation if we use W (4.114) with two boundary components instead. It will be like the calculation in Section [2.6.)

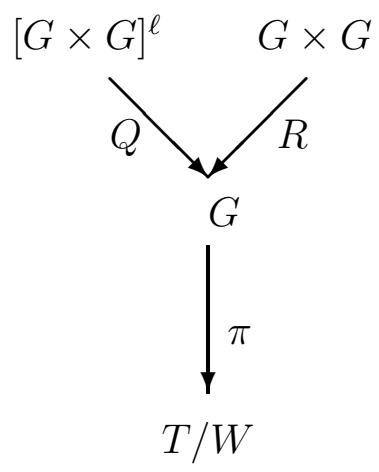


where

$$
Q\left(A_{1}, \cdots, A_{\ell}, B_{1}, \cdots, B_{\ell}\right)=\prod_{i=1}^{\ell} A_{i} B_{i} A_{i}^{-1} B_{i}^{-1}
$$

and $R\left(g_{1}, g_{2}\right)=g_{1} g_{2} g_{1}^{-1} g_{2}$. We have that the volume of the moduli space of flat connections on a once punctured Klein bottle with holonomy around the puncture given by $s$ is (by definition of the pushforward)

$$
\operatorname{Vol}(\mathcal{R}(K, g)) d g=R_{*}\left(d g_{1} \wedge d g_{2}\right)
$$

Moreover

$$
\operatorname{Vol}(\mathcal{R}(\Sigma))=\operatorname{Vol}\left(\mathcal{R}\left(\Sigma_{\ell} \sharp K\right)\right)=\int_{s \in G} d \operatorname{Viol}\left(\mathcal{R}\left(\Sigma_{\ell}, s\right)\right) \operatorname{Vol}(\mathcal{R}(K, s))
$$

Here we have used Lemma 6, which is again justified by Go1 Proposition 3.7.

Thus we have

$$
\begin{gathered}
\operatorname{Vol}(\mathcal{R}(\Sigma))=\int_{G} C_{1} \operatorname{VolT} \sum_{\alpha} \frac{1}{(\operatorname{dim}(\alpha))^{2 \ell-1}} \chi_{\alpha}(g) R_{*}\left(d g_{1} \wedge d g_{2}\right) \\
=C_{1} \operatorname{VolT} \sum_{\alpha} \frac{1}{(\operatorname{dim}(\alpha))^{2 \ell-1}} \int_{G \times G} \chi_{\alpha}\left(g_{1} g_{2} g_{1} g_{2}^{-1}\right) d g_{1} d g_{2} \\
=\frac{\sharp Z(G) \operatorname{Vol}(G)^{2 \ell}}{(2 \pi)^{(2 \ell-1)|G|-|T|}} \sum_{\alpha} \frac{1}{(\operatorname{dim}(\alpha))^{2 \ell-1}} \frac{1}{\operatorname{dim}(\alpha)} \int_{G} \chi_{\alpha}\left(g_{1}\right) \chi_{\alpha}\left(g_{1}\right) d g_{1}
\end{gathered}
$$

where we have used equation (51). Thus

$$
\operatorname{Vol}(\mathcal{R}(\Sigma))=\frac{\sharp Z(G) \operatorname{Vol}(G)^{2 \ell+1}}{(2 \pi)^{(2 \ell-1)|G|-|T|}} \sum_{\alpha=\bar{\alpha}} \frac{1}{(\operatorname{dim}(\alpha))^{2 \ell}}
$$

where we have used (cf: $[\mathrm{W}](4.50))$

$$
\int_{G} \chi_{\alpha}(g) \chi_{\alpha}(g) d g= \begin{cases}0 & \text { if } \alpha \neq \bar{\alpha} \\ \operatorname{Vol}(G) & \text { if } \alpha=\bar{\alpha}\end{cases}
$$

Thus our final formula is

$$
\operatorname{Vol}(\mathcal{R}(\Sigma))=\frac{\sharp Z(G) \operatorname{Vol}(G)^{2 \ell+1}}{(2 \pi)^{(2 \ell-1)|G|-|T|}} \sum_{\alpha=\bar{\alpha}} \frac{1}{(\operatorname{dim}(\alpha))^{2 \ell}}
$$

We compare our result with Witten's formula $[\mathrm{W}](4.77)$ :

$$
\operatorname{Vol}(\mathcal{R}(\Sigma))=\frac{\sharp Z(G) \operatorname{Vol}(G)^{2 \ell+1}}{(2 \pi)^{(2 \ell)|G|}} \sum_{\alpha=\bar{\alpha}} \frac{1}{(\operatorname{dim}(\alpha))^{2 \ell}}
$$

Our formula differs from Witten's by a multiplicative factor of $(2 \pi)^{|G|+|T|}$ because Witten chose a different normalization. 
This gives us a better idea of the geometric meaning of Witten's volume $[\mathrm{W}$. It is the integral of the measure derived from the symplectic volume on the moduli space of flat connections on an orientable surface and the pushforward volume of the Haar measure on products of $G$. Since the symplectic volume of the orientable part can also be explained as the Haar measure of the Lie group model (as we did in section 3.11), this explains why the Haar measure on products of Lie groups gives Witten's formula for the volume on the moduli space of flat connections on a nonorientable 2-manifold.

\section{Appendix A. Evaluation of An integral}

In this appendix we compute the integral (23). We assume $k$ is a positive integer, so

$$
\int_{\cos (\phi / 2)}^{1}\left(\frac{x}{l}-\sqrt{\left(\frac{x}{l}\right)^{2}-1}\right)^{2 k} \frac{d x}{\sqrt{1-x^{2}}}=S_{1}+S_{2}
$$

where

$$
S_{1}=\sum_{s=1}^{k} \frac{(2 k) !}{(2 s) !(2 k-2 s) !} \int_{\cos (\phi / 2)}^{1}\left(\frac{x}{l}\right)^{2 s}\left(\left(\frac{x}{l}\right)^{2}-1\right)^{k-s} \frac{d x}{\sqrt{1-x^{2}}}
$$

and

$$
S_{2}=-\sum_{s=0}^{k-1} \frac{(2 k) !}{(2 s+1) !(2 k-2 s-1) !} \int_{\cos \phi / 2}^{1}\left(\frac{x}{l}\right)^{2 s+1}\left(\left(\frac{x}{l}\right)^{2}-1\right)^{k-s-1} \sqrt{\left(\frac{x}{l}\right)^{2}-1} \frac{d x}{\sqrt{1-x^{2}}} .
$$

We can use the trigonometric substitution $x=\cos \theta$ for the integral (60). This yields

$$
\begin{gathered}
\int_{\cos (\phi / 2)}^{1} \sum_{s=1}^{k}\left(\frac{x^{2 s}}{l^{2 s}}\right)\left(\left(\frac{x}{l}\right)^{2}-1\right)^{k-s} \frac{d x}{\sqrt{1-x^{2}}} \\
=\int_{0}^{\phi / 2} \sum_{s=1}^{k} \frac{\left(\cos ^{2} \theta\right)^{s}}{l^{2 s}}\left(\frac{\cos ^{2} \theta}{l^{2}}-1\right)^{k-s} d \theta \\
=\sum_{s=1}^{k} \sum_{r=0}^{k-s} \frac{1}{l^{2(s+r)}}(-1)^{k-s-r} \frac{(k-s) !}{r !(k-s-r) !} \int_{0}^{\phi / 2}\left(\cos ^{2} \theta\right)^{s+r} d \theta \\
=\sum_{s=1}^{k} \sum_{r=0}^{k-s} \frac{1}{l^{2(s+r)}} \frac{(k-s) !(-1)^{k-s-r}}{r !(k-s-r) !}\left[\frac{1}{2^{s+r}} \frac{(2 s+2 r) !}{(s+r) !(s+r) !} \phi / 2\right.
\end{gathered}
$$




$$
\left.+\frac{1}{2^{2(s+r)-1}} \sum_{p=0}^{s+r-1} \frac{(2 s+2 r) !}{p !(2 s+2 r-2 p) !} \frac{\sin (s+r-p) \phi}{2(s+r-p)}\right]
$$

The integral (61) can be obtained by the substitution $y=\sqrt{1-x^{2}}$. Introducing $m^{2}=1-l^{2}$ and $y=m z$, this gives

$$
\begin{aligned}
& \int_{\cos (\phi / 2)}^{1} \sum_{s=0}^{k-1} \frac{(2 k) !}{(2 s+1) !(2 k-2 s-1) !}\left(\frac{x}{l}\right)^{2 s+1}\left(\left(\frac{x}{l}\right)^{2}-1\right)^{k-s-1} \sqrt{\left(\frac{x}{l}\right)^{2}-1} \frac{d x}{\sqrt{1-x^{2}}} \\
& =\sum_{s=0}^{k-1} \sum_{t=0}^{s} \int_{0}^{(1 / m) \sin (\phi / 2)} \frac{1}{l^{2 k}} \frac{s !}{t !(s-t) !}\left(-m^{2} z^{2}\right)^{t} m^{2(k-s-1)}\left(1-z^{2}\right)^{k-s-1} m^{2} \sqrt{1-z^{2}} d z
\end{aligned}
$$

Now let $z=\sin \theta$, and we obtain

$$
\begin{gathered}
\sum_{s=0}^{k-1} \sum_{t=0}^{s} \sum_{r=0}^{t} \frac{m^{2(k-s+t)}}{\left(1-m^{2}\right)^{k}}(-1)^{t+r} \frac{s !}{r !(t-r) !(s-t) !} \int_{0}^{\sin ^{-1}[(1 / m) \sin (\phi / 2)]}\left(\cos ^{2} \theta\right)^{k-s+r} d \theta \\
=\sum_{s=0}^{k-1} \sum_{t=0}^{s} \sum_{r=0}^{t} \frac{m^{2(k-(s-t))}}{\left(1-m^{2}\right)^{k}}(-1)^{t+r} \frac{s !}{r !(t-r) !(s-t) !} \times \\
\quad \times\left[\frac{1}{2^{2(k-(s-r))}} \frac{[2(k-s+r)] !}{(k-s+r) !(k-s+r) !} \theta_{m}\right. \\
\left.+\frac{1}{2^{2(k-s+r)-1}} \sum_{p=0}^{k-s+r-1} \frac{[2(k-s+r)] !}{p !(2[k-s+r]-p) !} \frac{\sin \left\{2 \theta_{m}(k-s+r-p)\right\}}{2(k-s+r-p)}\right]
\end{gathered}
$$

where $\theta_{m}=\arcsin (1 / m \sin (\phi / 2))$.

\section{REFERENCES}

[AMM] A. Alekseev, A. Malkin, and E. Meinrenken, Lie group valued moment maps, J. Differential Geom. 48 (1998), 445-495.

[AMW] A. Alekseev, E. Meinrenken, and C. Woodward, Duistermaat-Heckman measures and moduli spaces of flat bundles over surfaces, Geom. Funct. Anal. 12 (2002), no. 1, 1-31.

[AB] M.F. Atiyah and R. Bott, The Yang-Mills equations over Riemann surfaces, Philos. Trans. Roy. Soc. London Ser. A 308 (1983), no. 1505, 523615.

[AB2] M.F. Atiyah and R. Bott, The moment map and equivariant cohomology, Topology 23 (1984), no. 1, 1-28.

[BeSe] C. Becker and A. Sengupta, Sewing Yang-Mills measures and moduli spaces over compact surfaces. J. Funct. Anal. 52 (1998), 74-99.

[BGV] N. Berline, E. Getzler, M. Vergne, Heat Kernels and Dirac Operators. Springer-Verlag, New-York,

[BD] T. Bröcker and T. tom Dieck, Representations of Compact Lie Groups, Graduate Texts in Mathematics, 98. Springer-Verlag, New York, 1985. 
[B] P. Buser, Geometry and Spectra of Compact Riemann Surfaces, Birkhäuser (Progress in Math, Vol. 106), 1992.

[Cox] H.S.M. Coxeter, Introduction to Geometry, Wiley, New York, 1989.

[dC] M. do Carmo, Differential Geometry of Curves and Surfaces, Prentice-Hall, 1976.

[d'H] E. d'Hoker, String Theory, in Quantum Fields and Strings: A Course for Mathematicians (ed. P. Deligne et al.), Vol. 2, AMS, 1999, p. 807-1012.

[Fine] D. Fine, Quantum Yang-Mills on the two-sphere. Comm. Math. Phys. 134 (1990), 273-292.

[Fo] R. Forman, Small volume limits of 2-d Yang-Mills. Comm. Math. Phys. 151 (1993), 39-52.

[Fr] D. Freed, Reidemeister torsion, spectral sequences, and Brieskorn spheres. J. Reine Angew. Math. 429 (1992), 75-89.

[Go1] W.M. Goldman, The symplectic nature of fundamental groups of surfaces, Adv. Math. 54 (1984), 200-225.

[GM] W.M. Goldman and J.J. Millson, The deformation theory of representations of fundamental groups of compact Kähler manifolds, Bull. Amer. Math. Soc. (N.S.) 18 (1988), no. 2, 153-158.

[Liu] K. Liu, Heat kernel and moduli space, Math. Res. Lett. 3 (1996), 743-762.

[Ma] W. S. Massey, Algebraic Topology: An Introduction, Graduate Texts in Mathematics, 56. Springer-Verlag, New York, 1967.

[Mi] A.A. Migdal, Recursion equations in gauge field theories. Sov. Phys. JETP 42, 413-418 (1976).

[Se1] A. Sengupta, Yang-Mills on surfaces with boundary: quantum theory and symplectic limit. Commun. Math. Phys. 183 (1997) 661-705.

[Se2] A. Sengupta, Sewing symplectic volumes for flat connections over compact surfaces. J. Geom. Phys. 32 (2000) 269-292.

[Se3] A. Sengupta, The Yang-Mills measure and symplectic structure over spaces of connections. Quantization of singular symplectic quotients, 329-355, Birkhäuser (Progress in Mathematics vol. 198), 2001.

[Weis] Eric W. Weisstein, Spherical trigonometry. From MathWorld- A Wolfram Web Resource.

http: //mathworld. wolfram. com/SphericalTrigonometry.html

[W] E. Witten, On quantum gauge theories in two dimensions, Commun. Math. Phys. 141 (1991), 153-209

Department of Mathematics, National Cheng-Kung University, TaiWAN

E-mail address: nho@fields.utoronto.ca; nankuo@mail.ncku.edu.tw

Department of Mathematics, University of Toronto, Toronto, Ontario M5S 3G3, CANada

E-mail address: jeffrey@math.toronto.edu 